OPEN ACCESS

Edited by:

Sebastián A Riquelme, Columbia University Irving Medical

Center, United States

Reviewed by:

Frederick J Sheedy,

Trinity College Dublin, Ireland

Jianping Xie,

Southwest University, China

*Correspondence:

Lanbo Shi

shila@njms.rutgers.edu

Specialty section:

This article was submitted to

Microbial Immunology,

a section of the journal

Frontiers in Immunology

Received: 18 May 2021 Accepted: 25 June 2021

Published: 13 July 2021

Citation:

Jiang Q and Shi L (2021) Coordination

of the Uptake and Metabolism of

Amino Acids in Mycobacterium tuberculosis-Infected Macrophages.

Front. Immunol. 12:711462. doi: 10.3389/fimmu.2021.711462

\section{Coordination of the Uptake and Metabolism of Amino Acids in Mycobacterium tuberculosis- Infected Macrophages}

\author{
Qingkui Jiang and Lanbo Shi*
}

Public Health Research Institute, New Jersey Medical School, Rutgers Biomedical and Health Sciences, Rutgers The State University of New Jersey, Newark, NJ, United States

Macrophage polarization to the M1-like phenotype, which is critical for the proinflammatory and antimicrobial responses of macrophages against intracellular pathogens, is associated with metabolic reprogramming to the Warburg effect and a high output of NO from increased expression of NOS2. However, there is limited understanding about the uptake and metabolism of other amino acids during M1 polarization. Based on functional analysis of a group of upregulated transporters and enzymes involved in the uptake and/or metabolism of amino acids in Mycobacterium tuberculosis-infected macrophages, plus studies of immune cell activation, we postulate a coherent scheme for amino acid uptake and metabolism during macrophage polarization to the M1-like phenotype. We describe potential mechanisms that the increased arginine metabolism by NOS2 is metabolically coupled with system L transporters LAT1 and LAT2 for the uptake of neutral amino acids, including those that drive mTORC1 signaling toward the M1-like phenotype. We also discuss the underappreciated pleiotropic roles of glutamine metabolism in the metabolic reprogramming of $\mathrm{M} 1$-like macrophages. Collectively, our analyses argue that a coordinated amino acid uptake and metabolism constitutes an integral component of the broad metabolic scheme required for macrophage polarization to $\mathrm{M} 1$-like phenotype against $M$. tuberculosis infection. This idea could stimulate future experimental efforts to elucidate the metabolic map of macrophage activation for the development of anti-tuberculosis therapies.

Keywords: amino acid transporters, arginine metabolism, glutaminolysis, immunometabolism, M1 polarization, Mycobacterium tuberculosis, redox homeostasis, system L transporters

\section{INTRODUCTION}

Macrophage activation, in response to infection by intracellular pathogens or to treatment with lipopolysaccharide (LPS) and/or interferon- $\gamma$ (IFN- $\gamma$ ), results in M1-like or M1 phenotype characterized by high level-expression of pro-inflammatory and antimicrobial molecules (1-3). One of the key events accompanying M1 polarization is a programmed metabolic remodeling to the glycolysis pathway, reminiscent of the Warburg effect in cancer cells, to meet the increasing demand for 
energy, NADPH, and precursors for cell growth, differentiation and synthesis of effector molecules (4-6). This metabolic reprograming is an essential part of the defense mechanism of macrophages against infection by intracellular pathogens, such as Mycobacterium tuberculosis $(M t b)$ (7-9), the etiological agent of tuberculosis. Enhanced arginine uptake by the M1-like macrophage and its metabolism by the inducible nitric oxide synthase 2 (NOS2), which results in production of large amounts of NO, is indispensable for controlling many intracellular pathogens, including $M t b$ (10-12). Emerging evidence indicates that there is also an increased need for other amino acids, in particular those essential, for multiple cellular processes of activating immune cells, including energy metabolism, protein synthesis, redox balance, and cell growth and differentiation (13-16). For example, the uptake and metabolism of the essential neutral amino acids leucine and methionine are critical for clonal expansion and differentiation of effector $\mathrm{T}$ cells $(17,18)$. In addition, elevated tryptophan catabolism in macrophages due to $M t b$-induced expression of indoleamine 2,3dioxygenase 1 represses the ability of host immune cells to control the infection via inhibition of T cell proliferation (19). Despite this progress, our overall understanding of amino acid uptake mechanism and the contribution of their metabolism to the metabolic reprogramming required for macrophage polarization remains sketchy.

Membrane amino acid transporters mediate the uptake of amino acids from the extracellular milieu and/or their transfer between cellular compartments during immune cell activation (15). Abnormal expression and function of certain amino acid transporters are linked to a wide range of pathologies (20). Based on their sequence similarity or substrate specificity and transport mechanism, amino acid transporters are classified into various solute carrier (SLC) families or into various transport systems, respectively $(20,21)$. Among these, the SLC7 family comprises cationic amino acid transporters (CATs) of the $\mathrm{y}+$ system and members of the L-type amino acid transporter (LAT) system, the $y^{+} L$ system, and the $x_{c}^{-}$system (22). CATs mediate sodium-independent transport of cationic L-amino acids, such as arginine and lysine (23). Transporters of system $\mathrm{L}, \mathrm{y}+\mathrm{L}$, and $x_{c}^{-}$all function with a glycoprotein, $4 \mathrm{~F} 2 \mathrm{HC}$, encoded by $S L C 3 A 2 / S l c 3 a 2$, thereby forming the heteromeric amino acid transporters (HATs) $(24,25)$. HATs mediate exchange at a 1:1 stoichiometry with a broad spectrum of substrates that range from neutral amino acids [in the case of LAT1 (SLC7A5) and LAT2 (SLC7A8)] to negatively charged amino acids [in the case of the $x_{c}^{-}$system xCT (SLC7A11)]. Given that HATs require intracellularly accumulated amino acids and/or metabolites as export substrates for the exchange of amino acids from the extracellular milieu, their upregulation during immune cell activation suggests a close functional association of HATs with the metabolic program of activating immune cells. For example, LAT1mediated uptake of large, essential neutral amino acids, including leucine, is required for the metabolic reprogramming of effector $\mathrm{T}$ cells to support their proliferation and differentiation (18). Moreover, upregulated $x C T$ expression helps maintain redox balance and prolong the survival of activated macrophages by mitigating damage caused by oxidative stress (26). However, the expression and function of amino acid transporters in amino acid uptake and metabolism during macrophage polarization are still understudied.
Based on the biochemical properties of amino acid transporters and emerging evidence from transcriptomics and metabolomics studies of activation of bone marrow-derived macrophages (BMDMs), such as by infection with $M t b$ or with LPS treatment (27-30), we propose the idea that increased expression of several types of transporters and enzymes involved in the uptake and/or metabolism of amino acids are metabolically coordinated to support macrophage polarization toward the M1-like phenotype. We discuss potential mechanisms that a functional coupling between increased arginine metabolism and system L transporters (LAT1 and LAT2) drives the uptake of neutral amino acids, including those required for M1 polarization. We further discuss the role of glutamine metabolism in the metabolic reprogramming of M1like macrophages, which has usually been associated with M2 rather than with M1 macrophages.

\section{ARGININE UPTAKE, METABOLISM AND REGENERATION}

As a conditionally essential amino acid, arginine plays an important role in innate and adaptive immunity. Its uptake from the extracellular milieu, mediated by cationic amino acid transporter 2 (CAT2), a member of system y+ (31), functions as a critical regulator of immunity under inflammatory conditions $(32,33)$. Cat 2 deficiency is associated with poor macrophage activation phenotypes, resulting in decreased NO production from NOS2 in M1 macrophages, as well as reduced production of polyamines and proline by arginase 1 in M2 macrophages (12, 34). Moreover, during in vivo infection with pathogens that induce highly polarized Th2 or Th1 responses (33), CAT2 is a critical factor for the development of protective Th1-dependent immunity $(33,35)$, suggesting an important role of extracellular arginine for expression of host adaptive immunity.

Transcriptomics data from Mtb-infected BMDMs reveals a robust upregulation in the expression of Cat2 within $4-12$ hours of infection $(27-29,36)$. Since Cat2 upregulation is concurrent with the timing of M1-like polarization and upregulation of Nos2 (28, 29), as well as with metabolic reprogramming to the Warburg effect $(7,28,36-38)$, CAT2 may have an important role in $M t b$ induced M1-like polarization (Figure 1). This notion is supported by NO production from NOS2 of M1 macrophages or IFN- $\gamma$-stimulated BMDMs infected with $M$. bovis BCG coming predominantly from arginine taken up by CAT2, and accompaniment of active secretion of most of citrulline, the product of NOS2, into the extracellular milieu $(39,40)$ (Figure 1). During mycobacterial infection in vivo, the drastic increase of citrulline in infected lungs, which is associated with the expression of host adaptive immunity (41), is also consistent with the essential role of NOS2 in the mediation of the antimycobacterial response.

The importance of arginine in immune cell function is further supported by the observation that activated immune cells actively recycle citrulline for the regeneration of intracellular arginine. Citrulline recycling involves the induction of argininosuccinate synthetase 1 (ASS1), which catalyzes the 


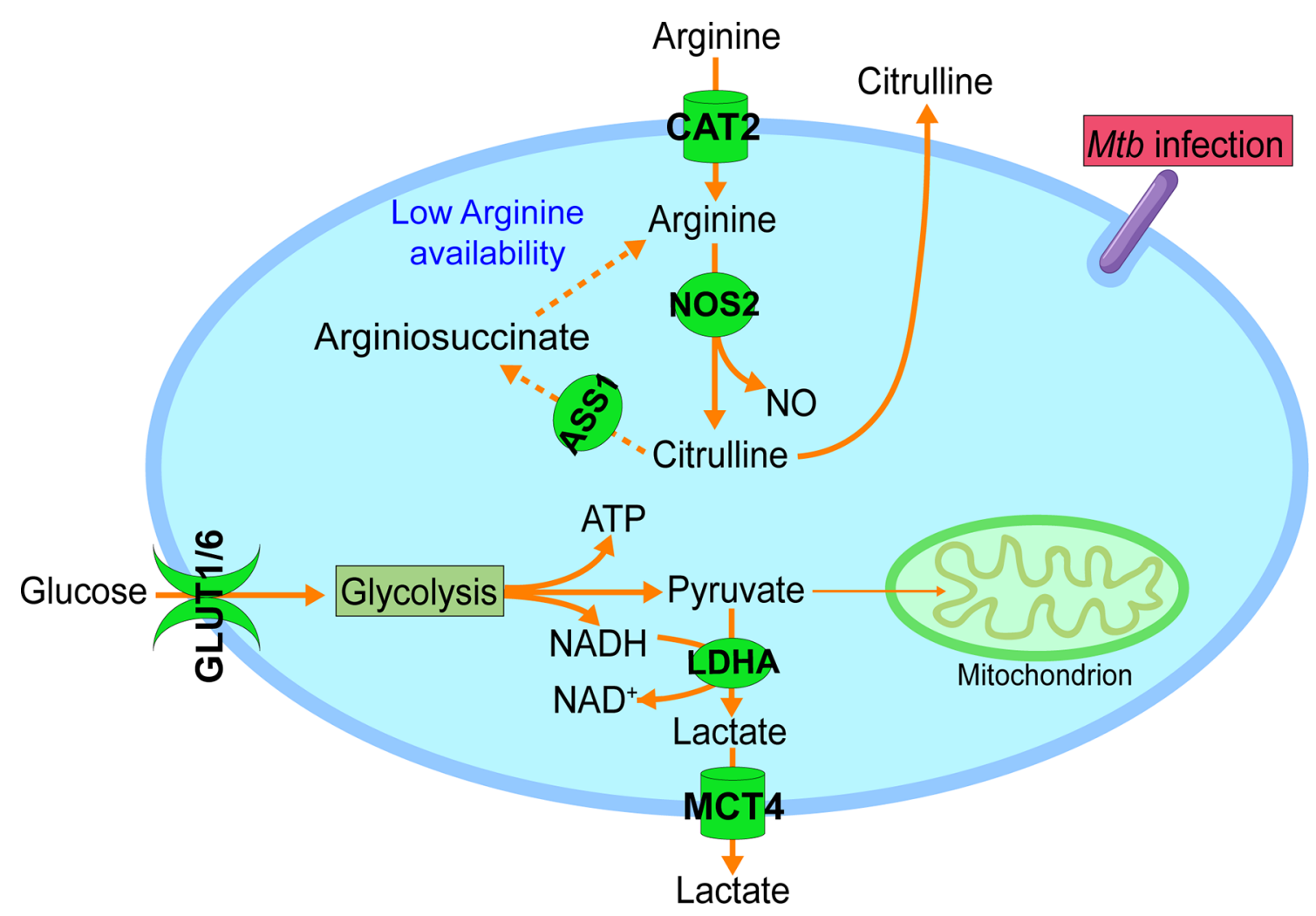

FIGURE 1 | Increased arginine uptake and metabolism during M1-like polarization. In addition to the metabolic reprogramming to glycolysis with increased glucose uptake (GLUT1 \& GLUT6) and lactate formation (LDHA) and secretion (MCT4), M1-like polarization is also accompanied by increased uptake and metabolism of arginine. Increased expression of cationic amino acid transporter 2 (CAT2) mediates the import of arginine from the extracellular microenvironment. Intracellular arginine is mainly catabolized by upregulated inducible nitric oxide synthetase 2 (NOS2) to NO and citrulline, and the latter is actively secreted to the extracellular microenvironment. With the decreasing availability of extracellular arginine, NOS2-derived citrulline is recycled for the regeneration of intracellular arginine via argininosuccinate synthetase 1 (ASS1) (dashed line), which will sustain the NO production by NOS2. GLUT1, glucose transporter 1; GLUT6, glucose transporter 6; LDHA, lactate dehydrogenase A; MCT4, monocarboxylate transporter 4 (MCT4). Data were derived from references and/or supplementary files therein (27-30).

formation of argininosuccinate from citrulline and aspartate. Argininosuccinate is further lysed by argininosuccinate lyase (ASL) for the production of intracellular arginine $(13,39,42)$. Under conditions of low arginine availability in the microenvironment, such as in infection foci, citrulline recycling is important for the antimycobacterial response by supporting effector $\mathrm{T}$ cell function and sustaining NO production from activated macrophages $(41,43)$. The upregulation of Ass 1 in $M t b$-infected BMDMs $(27,29)$, probably indicates decreasing availability of extracellular arginine as a consequence of early Cat2 induction, would thus warrant the supply of intracellular arginine for sustained NO production by NOS2 in activated macrophages (39) (Figure 1). This notion is supported by the observation that Ass1-deficient macrophages fail to salvage citrulline in arginine-scarce conditions, leading to their inability to control mycobacteria infection (39).

Increased LAT1 expression has been reported to be associated with citrulline transport to support the effector function of $\mathrm{T}$ cells (42). However, little is known about the metabolic and physiological roles of active transport of citrulline across the cell membrane in the context of macrophage polarization. Very interestingly, findings from a recent study using peritoneal macrophages have revealed additional roles of arginine metabolism in anti- $M t b$ macrophage function, which is related to mechanistic target of rapamycin (mTOR) activation and independent of NO production (44). In the following sections, we propose potential mechanisms by which uptake of essential neutral amino acids that are important for M1 polarization are metabolically coupled with the active production and secretion of citrulline to support macrophage polarization to the M1-like phenotype. We further discuss the involvement of glutamine metabolism in the metabolic reprogramming of M1like macrophages.

\section{UPTAKE OF NEUTRAL AMINO ACIDS BY LAT1 AND LAT2}

In addition to arginine, immune cell activation requires essential neutral amino acids for effector functions. Uptake of these amino acids is usually associated with increased expression of particular 
system $\mathrm{L}$ and/or $\mathrm{y}+\mathrm{L}$ transporters. The system $\mathrm{L}$ transporters LAT1 and LAT2 mediate obligatory $\mathrm{Na}^{+}$-independent neutral amino acid exchange with a 1:1 molar ratio (Figure 2). They have overlapping (but not identical) substrate selectivity, and their affinities for a given amino acid on the intracellular side of the cell membrane are much lower than those on the extracellular side $(25,45)$, thus favoring influx of their substrates. LAT1 has an apparent specificity for large neutral amino acids, including essential large, branched neutral amino acids, such as leucine and isoleucine (46). In contrast, LAT2 has broad substrate selectivity for both large and small neutral amino acids (47-49). In addition, these two LAT transporters have quite different apparent affinities for several amino acid substrates. For example, in contrast to LAT1, LAT2 has an apparent high affinity for glutamine and a low affinity of histidine (50). These findings underscore the potentially distinct roles of these two system L transporters for the uptake of particular neutral amino acids (Figure 2).

In $M t b$-infected BMDMs, concurrent with the upregulation of Cat2, the early induction of Slc7a5, Slc7a8 and Slc3a2 (27-29) suggests increased uptake of neutral amino acids by both LAT1/ 4F2hc and LAT2/4F2hc during M1-like polarization (Figure 2). LAT1-mediated uptake of the essential neutral amino acid leucine and its signaling are indispensable for activation of serine-threonine kinase complex mTORC1 and the subsequent metabolic reprogramming, clonal expansion, and effector

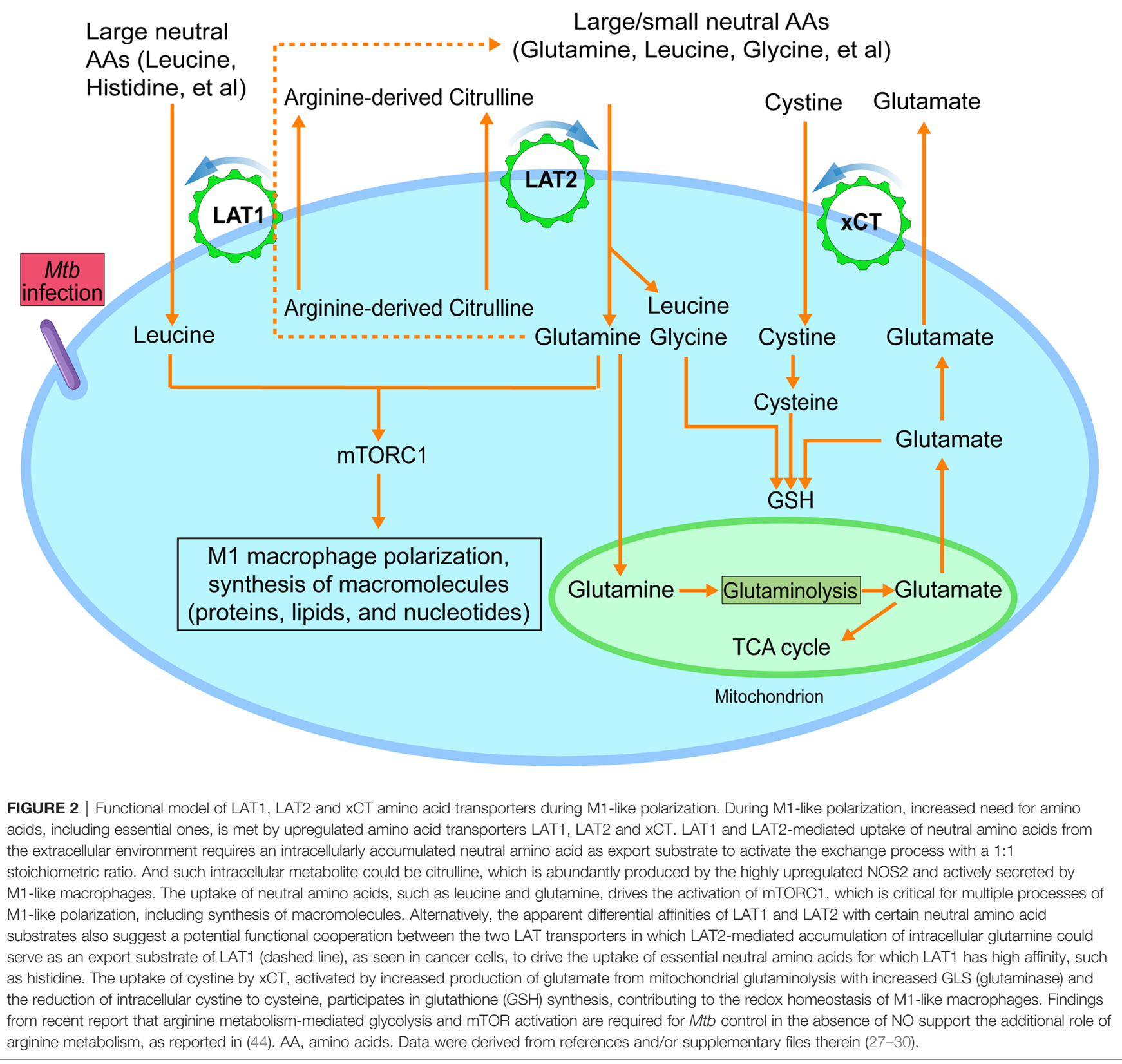


differentiation of activated T cells (18). Similarly, the LAT1mediated uptake of methionine, another essential neutral amino acid, functions as a main rate-limiting step during $\mathrm{T}$ cell activation for protein synthesis and the methionine cycle, which supplies methyl donors for the dynamic nucleotide methylation and epigenetic reprogram $(17,51)$. Still unknown are the specific amino acid substrates of LAT2 and their distinct functions in the metabolic reprogramming of immune cells. However, the apparent high affinity of LAT2 for certain amino acids, such as glutamine (50) plus its upregulation in $\mathrm{Mtb}$ infected BMDMs, suggest a potential role for LAT2 in the uptake of distinct neutral amino acids that are important for M1-like polarization. Thus, increased expression of both LAT1 and LAT2 during $M t b$ infection of macrophages would meet the demand of M1-like macrophages for various neutral amino acids, especially essential ones.

An open question regarding the operational mechanism of system $\mathrm{L}$ transporters is the identity of intracellularly accumulated neutral amino acids that are needed to activate the system $\mathrm{L}$ transporters by serving as their efflux substrate (45). Since LAT1-mediated citrulline transport is critical for the activation of effector $\mathrm{CD} 4^{+}$and $\mathrm{CD} 8^{+}$cells (42), it is plausible that citrulline production and its active secretion across the cell membrane of $M t b$ induced M1-like macrophages fulfill similar important metabolic functions during macrophage activation, as discussed in the following sections.

\section{XCT FOR REDOX HOMEOSTASIS}

Activation of innate and adaptive immune cells to the proinflammatory phenotype is associated with production of reactive oxygen and nitrogen species, molecules that are important for redox signaling and regulation of immune responses (52). Maintaining redox balance of activated immune cells is essential for preventing the deleterious effects of cytotoxic bioactive molecules. Synthesis of glutathione (GSH), a key small-molecule intracellular antioxidant, functions as one of the critical modulators of intracellular redox homeostasis by countering the increased levels of oxidative stress of activated immune cells $(26,53,54)$. GSH synthesis is associated with increased expression of $\mathrm{xCT}$, which, together with $4 \mathrm{~F} 2 \mathrm{hc}$, mediates $\mathrm{Na}^{+}$-independent and electroneutral import of extracellular anionic cystine with high affinity against export of intracellular glutamate (55) (Figure 2). The exchange is driven by intracellular reduction of cystine to cysteine and the high concentration of intracellular glutamate $(56,57)$. Cysteine, the reduced form of cystine, together with glutamate and glycine, participates in the synthesis of GSH (Figure 2).

$M t b$ infection of BMDMs leads to an increasing demand for GSH to protect activated macrophages from the cytotoxic bioactive molecules. Consequently, expression of genes encoding $\mathrm{xCT}$ for cystine import and enzymes involved in cystine reduction and glutathione synthesis is increased to meet this demand (36). As for the other substrates for GSH synthesis, glycine, a small neutral amino acid, can be imported by LAT2 (49), and it can also be produced from glycolysis intermediate 3-phosphoglycerate-derived intracellular serine
(58). Glutamate can come predominantly from the glutaminolysis pathway in mitochondria (Figure 2). This is consistent with the upregulation of Gls/GSL in $M t b$-infected BMDMs or human macrophages $(27,28,59)$, whose product glutaminase catalyzes a key rate-limiting step of glutaminolysis with the formation of glutamate. We also observed a rapid and extensive depletion of glutamine from the culture medium during the early phase of BMDM infection by $M t b$ (up to 12 hours post-infection, corresponding to the M1-like polarization) in comparison to a small amount of glutamate secreted into the culture medium (our unpublished data). The requirement of glutamine for the macrophage response to $M t b$ infection is further demonstrated by findings that human monocytederived macrophages (hMDMs) display decreased cytokine responses when cultured in medium devoid of glutamine or with the inhibition of glutamine utilization by glutaminasespecific inhibitors, such as BPTES and C968 (59).

As the most abundant and versatile amino acid in the body, glutamine is consumed by immune cells at a rate similar to or higher than glucose. Glutamine has been reported to be mainly associated with M2 macrophages based on transcriptional and metabolic profiling and analysis (60). However, the sampling and analysis that were carried out at or after $24 \mathrm{hr}$-treatment of BMDMs by LPS and IFN- $\gamma$ (for M1) or IL-4 (for M2) could have skewed the conclusion of glutamine metabolism toward an association with M2 polarization. This is because, as shown by transcriptional $(28,36)$ and metabolomics studies [supplemental data file of dynamic changes of metabolites during macrophage polarization in (30)], macrophage polarization to the M1-like phenotype, either induced by pathogen infection or by ligand signaling, such as by LPS and/or IFN- $\gamma$, occurs at up to $12 \mathrm{hrs}$ post-treatment or infection. Thus, analyses carried out at or beyond $24 \mathrm{hrs}$ post-treatment may not fully represent the immunometabolic state of M1-like but rather a transitioning state toward M2 macrophages, as evidenced by the expression of M2 markers (60). The different signaling pathways involving Mtb or LPS that resulted in differential immunometabolic properties of hMDMs, as reported (37), could be a contributing factor for the differential response in these cells. Involvement of glutamine in M1-like polarization is shown by increased enrichment of the tricarboxylic acid (TCA) cycle intermediate ${ }^{13} \mathrm{C} 4$ succinate from ${ }^{13} \mathrm{C} 5$ glutamine metabolomics study in LPS-activated BMDMs at 3 hrs post treatment (4); and glutamine-derived succinate functions as signaling molecules to promote the HIF- $1 \alpha$-mediated metabolic reprogramming of macrophages to glycolysis and proinflammation $(4,61)$. In proliferating cells, glutamine fulfills pleiotropic functions, including anaplerotic TCA cycle substrates, nucleotide synthesis, and synthesis of aspartate, to support cell growth and cellular redox homeostasis $(62,63)$. The observed increased dependence on glutamine in $M t b$-infected hMDMs or THP1 cells at 18 hrs p.i supports the involvement of glutamine in macrophage activation, while the contribution of glutamine to macrophage polarization to the M1, M2 or both was not clearly defined (64). The specific pathways involving glutamine metabolism contributing to the M1 and/or M2 polarization can be further dissected in future studies using therapeutic 
compounds, such as small molecule inhibitors BPTES, C968 and CB-839 targeting glutaminase $(59,62)$, and ${ }^{13} \mathrm{C}$ and ${ }^{15} \mathrm{~N}$ glutamine isotope tracing metabolomics.

\section{METABOLIC INTERPLAY BETWEEN ARGININE METABOLISM AND SYSTEM L TRANSPORTERS DURING M1-LIKE POLARIZATION}

Uptake of amino acids by various membrane transporters has been postulated to be a coordinated process in response to the metabolic and physiological demands of the cells. It is well known that apart from glucose, essential neutral amino acids are indispensable for cell growth and duplication during cell proliferation (65). Their uptake from the extracellular milieu is usually associated with increased expression of membrane transporters, including system L transporters and a neutral amino acid transporter SLC1A5 (66, 67). Functional coupling between LAT1 and SLC1A5 has been identified as a critical process for the unidirectional uptake of essential neutral amino acids required for cell growth and proliferation in cancer cells. Specifically, SLC1A5 mediates the influx of glutamine, which then activates the uptake of large essential amino acids, including leucine, by LAT1 by serving as its intracellular efflux substrate $(67,68)$. Consequently, the influx of large essential amino acids, such as leucine, together with glutamine, drives the mTORC1 activation and the metabolic reprogramming required for cell growth (68-70).

During $M t b$ infection, the downregulation of Slc1a5/SLC1A5 in both murine and human $(27,59)$ suggests that SLC1A5 may not be the major transporter for the uptake of glutamine in M1 macrophages. Instead, the increased expression of Slc7a8/Lat2 at early stage of macrophage infection from our analysis, the high affinity of LAT2 for glutamine (50), and the critical role of LAT2 in glutamine-dependent mTOR activation for the metabolic programming of cancer cells (71), suggest that LAT2 could be a main player in glutamine uptake by $M t b$ induced M1-like macrophages. Since LAT2 function requires an intracellularly accumulated neutral amino acid as an efflux substrate for the uptake of glutamine and other neutral amino acids, an ideal and metabolically suitable candidate that meets the criteria could be citrulline. This compound is made in abundant quantity by upregulated NOS2 and actively secreted to the extracellular milieu by M1 or M1-like macrophages $(39,40)$ [also refer to supplemental data in (30)]. The uptake of glutamine via LAT2-mediated citrulline/glutamine exchange could also activate LAT1 for the uptake of other large essential amino acids, especially those for which LAT1 has high affinity, by serving as its efflux substrate (Figure 2). This model of amino acid uptake via antiporters, such as system L transporters involving abundant glutamine for the uptake of essential amino acids, is consistent with the notion that glutamine serves as the cellular currency for the exchange (72). Another probable functional coupling scenario between arginine metabolism and system $\mathrm{L}$ transporters is that citrulline can also serve directly as the export substrate of LAT1 for the uptake of essential amino acids, given that citrulline has been identified as a substrate of LAT1 (42) (Figure 2). Thus, the working model for the functional coordination between arginine metabolism and system L transporters specifies that the activation of both LAT1 and LAT2 transporters by NOS2-derived citrulline, as an exchange substrate, results in the uptake of neutral amino acids, especially essential ones. This in turn leads to the activation of the mTORC1 pathway, such as by leucine and glutamine $(68,69)$ and metabolic reprogramming of M1-like macrophages (Figure 2). Indeed, the observations that arginine metabolism-mediated glycolysis and mTOR activation are required for $M t b$ control in the absence of $\mathrm{NO}$ support the additional role of arginine metabolism (44), as proposed in our model (Figure 2). Future studies combining genetics, metabolomics, and pharmacological approaches will be necessary to validate the proposed functional coupling of LAT transporters and arginine metabolism during M1 polarization. Since activated macrophages vigorously recycle citrulline when extracellular arginine level is low (43), it also will be interesting to investigate the specific role of these transporters in citrulline import in order to generate intracellular arginine.

\section{CONCLUDING REMARKS}

Our analyses suggest that functional cooperation among various amino acid transporters in the uptake and metabolism of amino acids constitutes an integral component of the metabolic remodel program of M1-like macrophages. In particular, the involvement of glutamine in the metabolic reprogramming during macrophage activation underscores the important role of glutamine as an important carbon and nitrogen source in M1-like macrophages. This immunometabolic switch accompanying M1-like polarization at early phase of host-pathogen interaction is in agreement with reduction of $M t b$ CFU in infected BMDMs (73). Thus, our understanding offers novel mechanistic insights into the broad metabolic remodeling program required for macrophage-mediated immunity, in addition to the known importance of the Warburg effect. However, our analysis doesn't address the mechanism by which $M t b$ modulates the immunometabolic switch required for the pro-inflammatory and antimicrobial response of macrophages to survive and/or persist in host cells, as shown in a recent study (74), given the limited studies in this rapidly evolving area of research. Further, given that different types of macrophages, in particular recruited $v s$. resident macrophages, are known to show variable degrees of immunometabolic responses to $M t b$ infection and/or stimuli $(8,74-78)$, and that macrophage response to infection and/ or stimuli show a dynamic defending and resolution/adaptation process $(28,36,64,79,80)$, cautions are warranted to interpret the immunometabolic programs of different macrophages at different stages of the infection and/or in responding to different stimuli. A similar metabolic coupling scenario in the uptake and metabolism of amino acids likely exists in in vivo settings, given that a similar set of genes, including Cat2, Nos2, Ass1, Lat1, Lat2, xCT and Gls was also upregulated in $M t b$-infected mouse lungs, along with the onset of host adaptive immunity (our unpublished observations). Such notion can be dissected in future studies in the context of the complex microenvironment of $M t b$-infected lungs. In particular, the necessity for glucose and other nutrients, including amino acids, required for 
the expression of antimicrobial and pro-inflammatory response likely varies among different immune cell types/subtypes in the microenvironment at different stages of the infection, which may manifest a fine-tuning of coordination and/or competition among nitrogen and carbon metabolic pathways of activated immune cells. These considerations should be taken into account to define the immunometabolic features of different immune cells in the in vivo settings. A better understanding of metabolic strategies of immune cell activation will lead to the development of immunometabolic therapeutics with enhanced efficacy against $M t b$ infection.

\section{DATA AVAILABILITY STATEMENT}

The gene expression data were derived from publicly available datasets deposited in the Genome Expression Omnibus (https:// www.ncbi.nlm.nih.gov/geo/) under accession numbers GSE31734 and GSE79733, and the supplementary data in (29).

\section{REFERENCES}

1. Mosser DM, Edwards JP. Exploring the Full Spectrum of Macrophage Activation. Nat Rev Immunol (2008) 8:958-69. doi: 10.1038/nri2448

2. Russell DG, Huang L. Immunometabolism at the Interface Between Macrophages and Pathogens. Nat Rev Immunol (2019) 19:291-304. doi: 10.1038/s41577-019-0124-9

3. Murray PJ, Wynn TA. Protective and Pathogenic Functions of Macrophage Subsets. Nat Rev Immunol (2011) 11:723-37. doi: 10.1038/nri3073

4. Tannahill GM, Curtis AM, Adamik J, Palsson-McDermott EM, McGettrick AF, Goel G, et al. Succinate is an Inflammatory Signal That Induces IL-1 Beta Through HIF-1 Alpha. Nature (2013) 496:238-42. doi: 10.1038/nature11986

5. Mills EL, Kelly B, Logan A, Costa AS, Varma M, Bryant CE, et al. Succinate Dehydrogenase Supports Metabolic Repurposing of Mitochondria to Drive Inflammatory Macrophages. Cell (2016) 167:457-70.e13. doi: 10.1016/ j.cell.2016.08.064

6. Palsson-McDermott EM, Curtis AM, Goel G, Lauterbach MA, Sheedy FJ, Gleeson LE, et al. Pyruvate Kinase M2 Regulates Hif-1alpha Activity and IL1beta Induction and is a Critical Determinant of the Warburg Effect in LPSActivated Macrophages. Cell Metab (2015) 21:65-80. doi: 10.1016/ j.cmet.2014.12.005

7. Osada-Oka M, Goda N, Saiga H, Yamamoto M, Takeda K, Ozeki Y, et al. Metabolic Adaptation to Glycolysis is a Basic Defense Mechanism of Macrophages for Mycobacterium Tuberculosis Infection. Int Immunol (2019) 31:781-93. doi: 10.1093/intimm/dxz048

8. Braverman J, Sogi KM, Benjamin D, Nomura DK, Stanley SA. HIF-1alpha Is an Essential Mediator of IFN-Gamma-Dependent Immunity to Mycobacterium Tuberculosis. J Immunol (2016) 197:1287-97. doi: 10.4049/ jimmunol.1600266

9. Gleeson LE, Sheedy FJ, Palsson-McDermott EM, Triglia D, O’Leary SM, O'Sullivan MP, et al. Cutting Edge: Mycobacterium Tuberculosis Induces Aerobic Glycolysis in Human Alveolar Macrophages That Is Required for Control of Intracellular Bacillary Replication. J Immunol (2016) 196:2444-9. doi: 10.4049/jimmunol.1501612

10. Nathan C, Shiloh MU. Reactive Oxygen and Nitrogen Intermediates in the Relationship Between Mammalian Hosts and Microbial Pathogens. Proc Natl Acad Sci USA (2000) 97:8841-8. doi: 10.1073/pnas.97.16.8841

11. MacMicking JD, North RJ, LaCourse R, Mudgett JS, Shah SK, Nathan CF. Identification of Nitric Oxide Synthase as a Protective Locus Against Tuberculosis. Proc Natl Acad Sci USA (1997) 94:5243-8. doi: 10.1073/ pnas.94.10.5243

12. Yeramian A, Martin L, Serrat N, Arpa L, Soler C, Bertran J, et al. Arginine Transport via Cationic Amino Acid Transporter 2 Plays a Critical Regulatory

\section{AUTHOR CONTRIBUTIONS}

LS conceived the concept and designed the manuscript outline. LS and QJ drafted the manuscript and prepared the figures. All authors contributed to the article and approved the submitted version.

\section{FUNDING}

The work was supported by National Institutes of Health (NIH) grant R01AI127844.

\section{ACKNOWLEDGMENTS}

We thank Karl Drlica and Carol Featherstone for critical reading of the manuscript.

Role in Classical or Alternative Activation of Macrophages. J Immunol (2006) 176:5918-24. doi: 10.4049/jimmunol.176.10.5918

13. Murray PJ. Amino Acid Auxotrophy as a System of Immunological Control Nodes. Nat Immunol 17 (2016) 17(2):132-9. doi: 10.1038/ni.3323

14. Li P, Yin YL, Li D, Kim SW, Wu G. Amino Acids and Immune Function. Br J Nutr (2007) 98:237-52. doi: 10.1017/S000711450769936X

15. Miyajima M. Amino Acids: Key Sources for Immunometabolites and Immunotransmitters. Int Immunol (2020) 32:435-46. doi: 10.1093/intimm/ dxaa019

16. McGaha TL, Huang L, Lemos H, Metz R, Mautino M, Prendergast GC, et al. Amino Acid Catabolism: A Pivotal Regulator of Innate and Adaptive Immunity. Immunol Rev (2012) 249:135-57. doi: 10.1111/j.1600-065X.2012.01149.x

17. Sinclair LV, Howden AJ. Antigen Receptor Control of Methionine Metabolism in T Cells. Elife (2019) 8:e44210. doi: 10.7554/eLife.44210

18. Sinclair LV, Rolf J, Emslie E, Shi YB, Taylor PM, Cantrell DA. Control of Amino-Acid Transport by Antigen Receptors Coordinates the Metabolic Reprogramming Essential for T Cell Differentiation. Nat Immunol (2013) 14:500-8. doi: 10.1038/ni.2556

19. Gautam US, Foreman TW, Bucsan AN, Veatch AV, Alvarez X, Adekambi T, et al. In Vivo Inhibition of Tryptophan Catabolism Reorganizes the Tuberculoma and Augments Immune-Mediated Control of Mycobacterium Tuberculosis. Proc Natl Acad Sci USA (2018) 115:E62-71. doi: 10.1073/pnas. 1711373114

20. Kandasamy P, Gyimesi G, Kanai Y, Hediger MA. Amino Acid Transporters Revisited: New Views in Health and Disease. Trends Biochem Sci (2018) 43:752-89. doi: 10.1016/j.tibs.2018.05.003

21. Poncet N, Taylor PM. The Role of Amino Acid Transporters in Nutrition. Curr Opin Clin Nutr Metab Care (2013) 16:57-65. doi: 10.1097/MCO. 0b013e32835a885c

22. Christensen HN. Role of Amino Acid Transport and Countertransport in Nutrition and Metabolism. Physiol Rev (1990) 70:43-77. doi: 10.1152/ physrev.1990.70.1.43

23. Closs EI, Boissel JP, Habermeier A, Rotmann A. Structure and Function of Cationic Amino Acid Transporters (CATs). J Membr Biol (2006) 213:67-77. doi: 10.1007/s00232-006-0875-7

24. Verrey F, Closs EI, Wagner CA, Palacin M, Endou H, Kanai Y. CATs and HATs: The SLC7 Family of Amino Acid Transporters. Pflugers Arch (2004) 447:532-42. doi: 10.1007/s00424-003-1086-Z

25. Fotiadis D, Kanai Y, Palacin M. The SLC3 and SLC7 Families of Amino Acid Transporters. Mol Aspects Med (2013) 34:139-58. doi: 10.1016/j.mam.2012.10.007

26. Nabeyama A, Kurita A, Asano K, Miyake Y, Yasuda T, Miura I, et al. xCT Deficiency Accelerates Chemically Induced Tumorigenesis. Proc Natl Acad Sci USA (2010) 107:6436-41. doi: 10.1073/pnas.0912827107 
27. Koo MS, Subbian S, Kaplan G. Strain Specific Transcriptional Response in Mycobacterium Tuberculosis Infected Macrophages. Cell Commun Signal (2012) 10:2. doi: 10.1186/1478-811X-10-2

28. Roy S, Schmeier S. Transcriptional Landscape of Mycobacterium Tuberculosis Infection in Macrophages. Sci Rep (2018) 8:6758. doi: 10.1038/s41598-01824509-6

29. Rothchild AC, Sissons JR, Shafiani S, Plaisier C, Min D, Mai D, et al. MiR-155Regulated Molecular Network Orchestrates Cell Fate in the Innate and Adaptive Immune Response to Mycobacterium Tuberculosis. Proc Natl Acad Sci USA (2016) 113:E6172-81. doi: 10.1073/pnas.1608255113

30. Peruzzotti-Jametti L, Bernstock JD, Vicario N, Costa ASH, Kwok CK, Leonardi T, et al. Macrophage-Derived Extracellular Succinate Licenses Neural Stem Cells to Suppress Chronic Neuroinflammation. Cell Stem Cell (2018) 22:355-68.e13. doi: 10.1016/j.stem.2018.01.020

31. MacLeod CL, Finley KD, Kakuda DK. Y(+)-Type Cationic Amino Acid Transport: Expression and Regulation of the mCAT Genes. J Exp Biol (1994) 196:109-21. doi: 10.1242/jeb.196.1.109

32. Closs EI, Scheld JS, Sharafi M, Förstermann U. Substrate Supply for NitricOxide Synthase in Macrophages and Endothelial Cells: Role of Cationic Amino Acid Transporters. Mol Pharmacol (2000) 57:68-74.

33. Thompson RW, Pesce JT, Ramalingam T, Wilson MS, White S, Cheever AW, et al. Cationic Amino Acid Transporter-2 Regulates Immunity by Modulating Arginase Activity. PloS Pathog (2008) 4:e1000023. doi: 10.1371/journal. ppat.1000023

34. Kakuda DK, Sweet MJ, Mac Leod CL, Hume DA, Markovich D. CAT2Mediated L-Arginine Transport and Nitric Oxide Production in Activated Macrophages. Biochem J (1999) 340(Pt 2):549-53. doi: 10.1042/bj3400549

35. Barry DP, Asim M, Scull BP, Piazuelo MB, de Sablet T, Lewis ND, et al. Cationic Amino Acid Transporter 2 Enhances Innate Immunity During Helicobacter Pylori Infection. PloS One (2011) 6:e29046. doi: 10.1371/ journal.pone.0029046

36. Shi L, Jiang Q, Bushkin Y, Subbian S. Biphasic Dynamics of Macrophage Immunometabolism During Mycobacterium Tuberculosis Infection. mBio (2019) 10:e2550-18. doi: 10.1128/mBio.02550-18

37. Lachmandas E, Beigier-Bompadre M, Cheng SC, Kumar V, van Laarhoven A, Wang X, et al. Rewiring Cellular Metabolism via the AKT/mTOR Pathway Contributes to Host Defence Against Mycobacterium Tuberculosis in Human and Murine Cells. Eur J Immunol (2016) 46:2574-86. doi: 10.1002/ eji.201546259

38. Shi L, Eugenin EA, Subbian S. Immunometabolism in Tuberculosis. Front Immunol (2016) 7:150. doi: 10.3389/fimmu.2016.00150

39. Qualls JE, Subramanian C, Rafi W, Smith AM, Balouzian L, DeFreitas AA, et al. Sustained Generation of Nitric Oxide and Control of Mycobacterial Infection Requires Argininosuccinate Synthase 1. Cell Host Microbe (2012) 12:313-23. doi: 10.1016/j.chom.2012.07.012

40. Benninghoff B, Lehmann V, Eck HP, Droge W. Production of Citrulline and Ornithine by Interferon-Gamma Treated Macrophages. Int Immunol (1991) 3:413-7. doi: $10.1093 /$ intimm/3.5.413

41. Lange SM, McKell MC, Schmidt SM, Zhao J. L-Arginine Synthesis From LCitrulline in Myeloid Cells Drives Host Defense Against Mycobacteria In Vivo. J Immunol (2019) 202:1747-54. doi: 10.4049/jimmunol.1801569

42. Werner A, Koschke M, Leuchtner N, Luckner-Minden C, Habermeier A, Rupp J, et al. Reconstitution of $\mathrm{T}$ Cell Proliferation Under Arginine Limitation: Activated Human T Cells Take Up Citrulline via L-Type Amino Acid Transporter 1 and Use It to Regenerate Arginine After Induction of Argininosuccinate Synthase Expression. Front Immunol (2017) 8:864-4. doi: 10.3389/fimmu.2017.00864

43. Lange SM, McKell MC, Schmidt SM, Hossfeld AP, Chaturvedi V, Kinder JM, et al. L-Citrulline Metabolism in Mice Augments CD4+ T Cell Proliferation and Cytokine Production In Vitro, and Accumulation in the MycobacteriaInfected Lung. Front Immunol (2017) 8:1651. doi: 10.3389/fimmu.2017.01561

44. McKell MC, Crowther RR, Schmidt SM, Robillard MC, Cantrell R, Lehn MA, et al. Promotion of Anti-Tuberculosis Macrophage Activity by L-Arginine in the Absence of Nitric Oxide. Front Immunol (2021) 12:653571. doi: 10.3389/ fimmu.2021.653571

45. Meier C, Ristic Z, Klauser S, Verrey F. Activation of System L Heterodimeric Amino Acid Exchangers by Intracellular Substrates. EMBO J (2002) 21:580-9. doi: 10.1093/emboj/21.4.580
46. Kanai Y, Segawa H, Miyamoto K, Uchino H, Takeda E, Endou H. Expression Cloning and Characterization of a Transporter for Large Neutral Amino Acids Activated by the Heavy Chain of 4F2 Antigen (CD98). J Biol Chem (1998) 273:23629-32. doi: 10.1074/jbc.273.37.23629

47. Wang Q, Holst J. L-Type Amino Acid Transport and Cancer: Targeting the Mtorcl Pathway to Inhibit Neoplasia. Am J Cancer Res (2015) 5:1281-94.

48. Pineda M, Fernandez E, Torrents D, Estevez R, Lopez C, Camps M, et al. Identification of a Membrane Protein, LAT-2, That Co-Expresses With 4F2 Heavy Chain, an L-Type Amino Acid Transport Activity With Broad Specificity for Small and Large Zwitterionic Amino Acids. J Biol Chem (1999) 274:19738-44. doi: 10.1074/jbc.274.28.19738

49. Segawa H, Fukasawa Y, Miyamoto K, Takeda E, Endou H, Kanai Y. Identification and Functional Characterization of a Na+-Independent Neutral Amino Acid Transporter With Broad Substrate Selectivity. J Biol Chem (1999) 274:19745-51. doi: 10.1074/jbc.274.28.19745

50. Rossier G, Meier C, Bauch C, Summa V, Sordat B, Verrey F, et al. LAT2, a New Basolateral 4f2hc/CD98-Associated Amino Acid Transporter of Kidney and Intestine. J Biol Chem (1999) 274:34948-54. doi: 10.1074/jbc.274.49.34948

51. Roy DG, Chen J, Mamane V, Ma EH, Muhire BM, Sheldon RD, et al. Methionine Metabolism Shapes T Helper Cell Responses Through Regulation of Epigenetic Reprogramming. Cell Metab (2020) 31:250-66.e9. doi: 10.1016/j.cmet.2020.01.006

52. Sun L, Wang X, Saredy J, Yuan Z, Yang X, Wang H. Innate-Adaptive Immunity Interplay and Redox Regulation in Immune Response. Redox Biol (2020) 37:101759. doi: 10.1016/j.redox.2020.101759

53. Lian G, Gnanaprakasam JNR, Wang T, Wu R, Chen X, Liu L, et al. Glutathione De Novo Synthesis But Not Recycling Process Coordinates With Glutamine Catabolism to Control Redox Homeostasis and Directs Murine T Cell Differentiation. eLife (2018) 7:e36158. doi: 10.7554/eLife.36158

54. Cai Y, Yang Q, Liao M, Wang H, Zhang C, Nambi S, et al. xCT Increases Tuberculosis Susceptibility by Regulating Antimicrobial Function and Inflammation. Oncotarget (2016) 7:31001-13. doi: 10.18632/oncotarget.9052

55. Sato H, Tamba M, Ishii T, Bannai S. Cloning and Expression of a Plasma Membrane Cystine/Glutamate Exchange Transporter Composed of Two Distinct Proteins. J Biol Chem (1999) 274:11455-8. doi: 10.1074/jbc.274.17.11455

56. Lewerenz J, Hewett SJ, Huang Y, Lambros M, Gout PW, Kalivas PW, et al. The Cystine/Glutamate Antiporter System X(C)(-) in Health and Disease: From Molecular Mechanisms to Novel Therapeutic Opportunities. Antioxidants Redox Signaling (2013) 18:522-55. doi: 10.1089/ars.2011.4391

57. Lu SC. Regulation of Glutathione Synthesis. Mol Aspects Med (2009) 30:4259. doi: 10.1016/j.mam.2008.05.005

58. Rodriguez AE, Ducker GS, Billingham LK, Martinez CA, Mainolfi N, Suri V, et al. Serine Metabolism Supports Macrophage IL-1 $\beta$ Production. Cell Metab (2019) 29:1003-11.e4. doi: 10.1016/j.cmet.2019.01.014

59. Koeken V, Lachmandas E, Riza A, Matzaraki V, Li Y, Kumar V, et al. Role of Glutamine Metabolism in Host Defense Against Mycobacterium Tuberculosis Infection. J Infect Dis (2019) 219:1662-70. doi: 10.1093/infdis/jiy709

60. Jha Abhishek K., Huang Stanley C.-C., Sergushichev A, Lampropoulou V, Ivanova Y, Loginicheva E, et al. Network Integration of Parallel Metabolic and Transcriptional Data Reveals Metabolic Modules That Regulate Macrophage Polarization. Immunity (2015) 42:419-30. doi: 10.1016/j.immuni.2015.02.005

61. Mills E, O'Neill LA. Succinate: A Metabolic Signal in Inflammation. Trends Cell Biol (2014) 24:313-20. doi: 10.1016/j.tcb.2013.11.008

62. Alkan HF, Walter KE, Luengo A, Madreiter-Sokolowski CT, Stryeck S, Lau AN, et al. Cytosolic Aspartate Availability Determines Cell Survival When Glutamine Is Limiting. Cell Metab (2018) 28:706-720.e6. doi: 10.1016/j.cmet.2018.07.021

63. Gross MI, Demo SD, Dennison JB, Chen L, Chernov-Rogan T, Goyal B, et al. Antitumor Activity of the Glutaminase Inhibitor CB-839 in Triple-Negative Breast Cancer. Mol Cancer Ther (2014) 13:890-901. doi: 10.1158/15357163.MCT-13-0870

64. Cumming BM, Addicott KW, Adamson JH, Steyn AJ. Mycobacterium Tuberculosis Induces Decelerated Bioenergetic Metabolism in Human Macrophages. Elife (2018) 7:e39169. doi: 10.7554/eLife.39169

65. Zhu J, Thompson CB. Metabolic Regulation of Cell Growth and Proliferation. Nat Rev Mol Cell Biol (2019) 20:436-50. doi: 10.1038/s41580-019-0123-5

66. Häfliger P, Charles R-P. The L-Type Amino Acid Transporter LAT1-An Emerging Target in Cancer. Int J Mol Sci (2019) 20:2428. doi: 10.3390/ ijms20102428 
67. Fuchs BC, Bode BP. Amino Acid Transporters ASCT2 and LAT1 in Cancer: Partners in Crime? Semin Cancer Biol (2005) 15:254-66. doi: 10.1016/ j.semcancer.2005.04.005

68. Nicklin P, Bergman P, Zhang B, Triantafellow E, Wang H, Nyfeler B, et al. Bidirectional Transport of Amino Acids Regulates mTOR and Autophagy. Cell (2009) 136:521-34. doi: 10.1016/j.cell.2008.11.044

69. Jewell JL, Kim YC, Russell RC, Yu FX, Park HW, Plouffe SW, et al. Metabolism. Differential Regulation of Mtorc1 by Leucine and Glutamine. Science (2015) 347:194-8. doi: 10.1126/science.1259472

70. Shimobayashi M, Hall MN. Multiple Amino Acid Sensing Inputs to Mtorc1. Cell Res (2016) 26:7-20. doi: 10.1038/cr.2015.146

71. Feng M, Xiong G, Cao Z, Yang G, Zheng S, Qiu J, et al. LAT2 Regulates Glutamine-Dependent mTOR Activation to Promote Glycolysis and Chemoresistance in Pancreatic Cancer. J Exp Clin Cancer Res (2018) 37:274. doi: 10.1186/s13046-018-0947-4

72. Bröer S. Adaptation of Plasma Membrane Amino Acid Transport Mechanisms to Physiological Demands. Pflugers Arch (2002) 444:457-66. doi: 10.1007/s00424-002-0840-y

73. Rohde KH, Veiga DF, Caldwell S, Balazsi G, Russell DG. Linking the Transcriptional Profiles and the Physiological States of Mycobacterium Tuberculosis During an Extended Intracellular Infection. PloS Pathog (2012) 8:e1002769. doi: 10.1371/journal.ppat.1002769

74. Hackett EE, Charles-Messance H, O'Leary SM, Gleeson LE, Muñoz-Wolf N, Case S, et al. Mycobacterium Tuberculosis Limits Host Glycolysis and IL-1 $\beta$ by Restriction of PFK-M via MicroRNA-21. Cell Rep (2020) 30:124-36.e4. doi: 10.1016/j.celrep.2019.12.015

75. Sivangala Thandi R, Radhakrishnan RK, Tripathi D, Paidipally P, Azad AK, Schlesinger LS, et al. Ornithine-A Urea Cycle Metabolite Enhances Autophagy and Controls Mycobacterium Tuberculosis Infection. Nat Commun (2020) 11:3535. doi: 10.1038/s41467-020-17310-5
76. Woods PS, Kimmig LM, Meliton AY, Sun KA, Tian Y, O'Leary EM, et al Tissue-Resident Alveolar Macrophages Do Not Rely on Glycolysis for LPSInduced Inflammation. Am J Respir Cell Mol Biol (2020) 62:243-55. doi: 10.1165/rcmb.2019-0244OC

77. Svedberg FR, Brown SL, Krauss MZ, Campbell L, Sharpe C, Clausen M, et al. The Lung Environment Controls Alveolar Macrophage Metabolism and Responsiveness in Type 2 Inflammation. Nat Immunol (2019) 20:571-80. doi: 10.1038/s41590-019-0352-y

78. Huang L, Nazarova EV, Tan S, Liu Y, Russell DG. Growth of Mycobacterium Tuberculosis In Vivo Segregates With Host Macrophage Metabolism and Ontogeny. J Exp Med (2018) 215:1135-52. doi: 10.1084/jem.20172020

79. Liu TF, Vachharajani VT, Yoza BK, McCall CE. NAD+-Dependent Sirtuin 1 and 6 Proteins Coordinate a Switch From Glucose to Fatty Acid Oxidation During the Acute Inflammatory Response. J Biol Chem (2012) 287:25758-69. doi: 10.1074/jbc.M112.362343

80. Moreira D, Rodrigues V, Abengozar M, Rivas L, Rial E, Laforge M, et al. Leishmania Infantum Modulates Host Macrophage Mitochondrial Metabolism by Hijacking the SIRT1-AMPK Axis. PloS Pathog (2015) 11: e1004684. doi: 10.1371/journal.ppat.1004684

Conflict of Interest: The authors declare that the research was conducted in the absence of any commercial or financial relationships that could be construed as a potential conflict of interest.

Copyright (C) 2021 Jiang and Shi. This is an open-access article distributed under the terms of the Creative Commons Attribution License (CC BY). The use, distribution or reproduction in other forums is permitted, provided the original author(s) and the copyright owner(s) are credited and that the original publication in this journal is cited, in accordance with accepted academic practice. No use, distribution or reproduction is permitted which does not comply with these terms. 